\title{
Acute pancreatitis in pregnant woman: case report
}

Keywords: acute pancreatitis, primigravida, endoscopic retrograde cholangiopancreatography

\section{Case report}

This article describes a case of acute pancreatitis in a 36yearsold primigravida woman who underwent endoscopic retrograde cholangiopancreatography (ERCP). At 27weeks+4days of gestation she was admitted in our clinic for severe abdominal pain, nausea, vomiting, and heart rhythm disorders. She was without risk factors for acute pancreatitis, but at a blood test she had abnormal values of WBC (14.40 x10^3/ul), PCR (14.44mg/L), $\gamma$-GT (66IU/L), ASTGOT (73IU/L),ALT-GPT (84 IU/L), LDH (387IU/L), pancreatic amylase $(1811 \mathrm{UI} / \mathrm{L})$, lipase $(4485 \mathrm{IU} / \mathrm{L})$, total $(1.68 \mathrm{mg} / \mathrm{dL})$, and direct $(1.41 \mathrm{mg} / \mathrm{dL})$ bilirubin. Parenteral nutrition, pain therapy, fluid and electrolyte support and antibiotic coverage were immediately initiated. Abdominal ultrasound showed some small gallstones and biliary sludge. After multidisciplinary consultation a ERCP associated with biliary sphincterotomy and drainage of the gallbladder sludge was performed. In the early postoperative period the patient developed progressive pulmonary desaturation with concomitant bradycardia and heart rhythm disorders. Angio-CT showed lower lobar pneumonia. The patient had no fever and analyses for Legionella, Pneumococcus and Aspergillus were negative. She received ventilation and antibiotic prophylaxis in order to prevent superimposed infections. She gradually recovered and after normalization of clinical and biochemical parameters she was discharged at 31 weeks of gestation. Subsequently, she was re-admitted at 36 weeks of gestation for suspected recurrence of pancreatitis. Instead, preeclampsia was diagnosed and labor induced at 38 weeks of gestation. The woman delivered vaginally a male infant, weighing 2.385grams with an Apgar score of 9/10.

Pregnancy may increase the risk of gallstones because of the estrogen-induced increase of cholesterol secretion, and the progesterone-induced reduction of bile acid secretion and gallbladder emptying. ${ }^{1}$ In spite of that, acute pancreatitis is rarely observed during

\author{
Volume 4 Issue I - 2018 \\ Baccarini G, Filip G, Driul L, Cagnacci A \\ University of Udine, Italy
}

Correspondence: Driul L, University of Udine, Italy, Email lorenza.driul@uniud.it

Received: January 10, 2018| Published: February 16, 2018

pregnancy. In a retrospective cohort study of 16,000 deliveries, only eight cases were signaled, of which five due to gallstones and three idiopathic. ${ }^{2}$ Even when it occurs, pancreatitis is rarely complicated during pregnancy. In a retrospective cohort study on 96 pregnant patients with acute pancreatitis, 96percent did not show complications. ${ }^{3}$ Ours is one of the few cases in which an instrumental procedure was necessary to obtain resolution. Besides the associated complications at the end, the case was successfully managed.

\section{Acknowledgements}

None.

\section{Conflict of interest}

Author declares that there is no conflict of interest.

\section{References}

1. David C Brooks. Gallstones in pregnancy. 2017.

2. Jouppila P, Mokka R, Larmi TK. Acute pancreatitis in pregnancy. Surg Gynecol Obstet. 1974.

3. Tang SJ, Rodriguez-Frias E, Singh S. Acute pancreatitis during pregnancy. Clin Gastroenterol Hepatol. 2010;8(1):85-90. 